\title{
SIMULATING NANOSCALE SEMICONDUCTOR DEVICES
}

\author{
M. S. LASATER \\ Center for Research in Scientific Computing, Department of Mathematics, North Carolina State University, \\ Raleigh, North Carolina State University 27695-8205, USA \\ mslasate@unity.ncsu.edu \\ C. T. KELLEY
}

Center for Research in Scientific Computing, Department of Mathematics, North Carolina State University, Raleigh, North Carolina State University 27695-8205, USA

tim_kelley@ncsu.edu

\section{A. G. SALINGER}

Sandia National Laboratories, P. O. Box 5800, MS-1111, Albuquerque, New Mexico 87185, USA

agsalin@sandia.gov

\section{L. WOOLARD}

U. S. Army Research Office, U. S. Army Research Laboratory, RTP, North Carolina 27709-2211, USA

dwight.woolard@us.army.mil

\section{P. ZHAO}

Electrical and Computer Engineering Department, North Carolina State University, Raleigh, North Carolina 27695-8205, USA

pzhao@eos.ncsu.edu

The next generation of electronic devices will be developed at the nanoscale and molecular level, where quantum mechanical effects are observed. These effects must be accounted for in the design process for such small devices. One prototypical nanoscale semiconductor device under investigation is a resonant tunneling diode (RTD). Scientists are hopeful the quantum tunneling effects present in an RTD can be exploited to induce and sustain $\mathrm{THz}$ frequency current oscillations. To simulate the electron transport within the RTD, the Wigner-Poisson equations are used. These equations describe the time evolution of the electrons' distribution within the device. In this paper, this model and a parameter study using this model will be presented. The parameter study involves calculating the steady-state current output from the RTD as a function of an applied voltage drop across the RTD and also calculating the stability of that solution. To implement the parameter study, the computational model was connected to LOCA (Library of Continuation Algorithms), a part of Sandia National Laboratories parallel solver project, Trilinos. Numerical results will be presented.

Keywords: Resonant tunneling diodes; continuation methods; Hopf bifurcation. 


\section{Introduction}

In the next few decades, if the trend predicted by Moore's Law ${ }^{1}$ is to continue, semiconductor devices will need to be scaled down to the nanoscale. The principal hindrance in the design of these small devices will be that quantum physics, instead of classical physics, will dictate their operation. Physicists and engineers do not yet fully understand what new features will be present due to quantum effects. This lack of knowledge motivates deriving an accurate model of electron transport at the quantum level. One such model, the Wigner-Poisson equations ${ }^{2}$, is utilized in this paper for simulating electron transport in a prototypical nanoscale semiconductor device, the resonant tunneling diode (RTD).

The RTD has been extensively researched in the past two decades ${ }^{3,4,5}$. In the 1980s, these devices were placed in circuit configurations as gain elements to induce a highfrequency current oscillation ${ }^{6}$. While high-frequency oscillations developed (highfrequency here means $\mathrm{THz}$ ), oscillations at lower frequency modes also developed, and too much power was lost at these lower frequency oscillations. To prohibit these lower frequency oscillations from developing, the device size had to be further scaled down, but this also led to reduced power output from the high frequency oscillation ${ }^{7}$. Ongoing research with the RTD involves removing it from the circuit to see if some intrinsic mechanism can be exploited to created $\mathrm{THz}$ frequency oscillations.

Figure 1 presents a schematic of an RTD and the diagram of the electric potential within the RTD when a large voltage drop $V$ is applied to the RTD. An RTD is made by growing alternating layers of semiconductor materials, denoted by material I and material II in Figure 1. The difference between the materials is that material II has a larger bandgap than material I. This difference causes the discontinuous jumps in the electric potential present in Figure 1. For our simulations, material I is gallium arsenide (GaAs), and material II is aluminum gallium arsenide (AlGaAs).

At the left and right ends of the RTD, there is doping, where atoms that have more electrons than the semiconductor is placed in the device to increase the number of electrons present in the device. The voltage drop across the device drives electrons to the right side of the RTD, creating a current. Since in quantum physics, electrons are treated as waves instead of particles, when they encounter the potential barriers, they have the ability to tunnel through them and reach the right side of the RTD. This quantum tunneling effect is the basis of the RTD's operation, and scientist hope this mechanism can be understood and controlled by using the Wigner-Poisson simulations in order to create and sustain $\mathrm{THz}$ frequency current oscillation within the RTD.

\section{The Wigner-Poisson Equations}

The Wigner-Poisson equations describe the time-evolution of the electron distribution within the RTD. The equations consist of a nonlinear integro-partial differential equation (IPDE) coupled with Poisson's equation to determine the electrostatic potential within the device. The IPDE is given by 


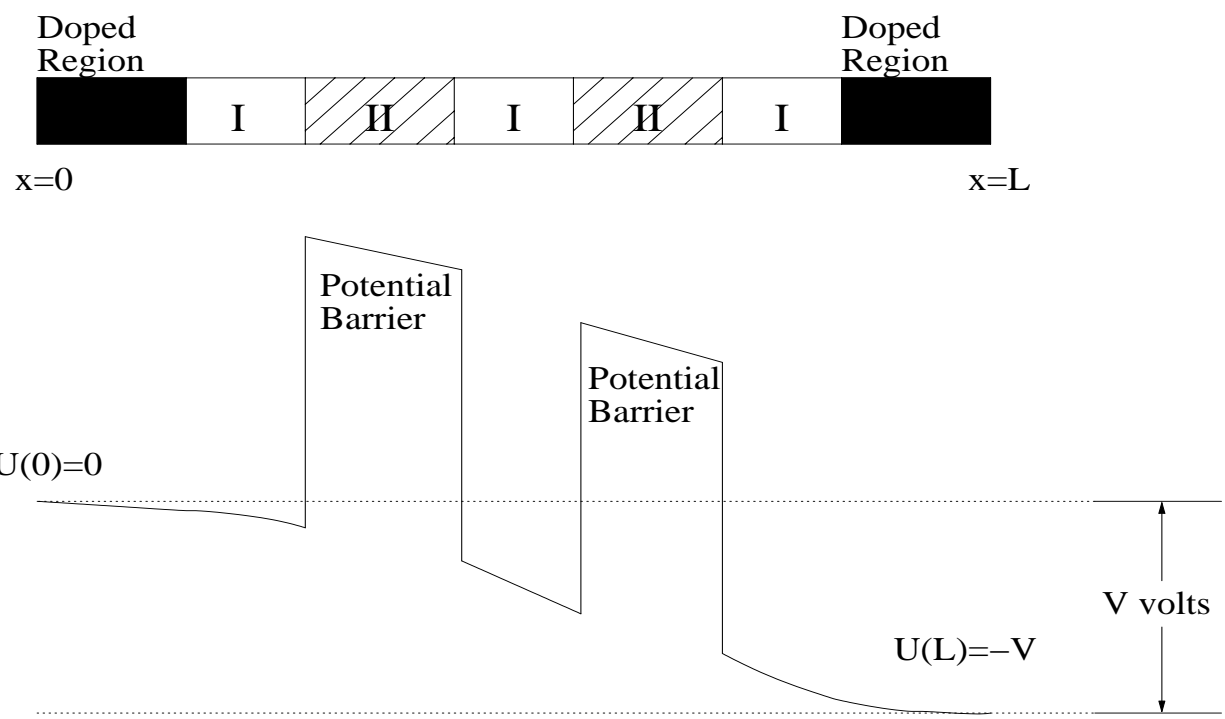

Fig. 1. Diagram of an RTD and the electric potential within the RTD

$$
\partial f / \partial t=W(f)=K f+P(f)+S(f) .
$$

Here, $f=f(x, k, t)$ is the electrons' distribution within the RTD. It is a function of the electron's position $x$, where $x$ is in $[0, L]$ ( $L$ being the length of the RTD), electron's momentum $k$, where $k$ is in $(-\infty, \infty)$, and time $t>0$. Given an initial electron distribution, Eq. (1) is used to track how the distribution changes in time. This type of simulation is discussed in the time-dependent simulation section.

The second type of simulation we perform involves directly finding steady-state electron distributions. These distributions will satisfy the equation

$$
W(f)=K f+P(f)+S(f)=0 .
$$

This type of simulation is discussed in the steady-state simulation section.

The first term in the equation, $K f$, is a linear term and represents the kinetic energy effects on the distribution. It is given by

$$
K f=-h k / 2 \pi m^{*} \partial f / \partial x .
$$

In this term, $h$ is Planck's constant, and $m^{*}$ is the electron's effective mass.

The second term in the equation, $P(f)$, represents the potential energy effects on the distribution and is the nonlinear term in the equation. It is given by 


$$
P(f)=-4 / h \int_{-\infty}^{\infty} f\left(x, k^{\prime}\right) T\left(x, k-k^{\prime}\right) d k^{\prime} .
$$

The kernel function, $T(x, k)$, which $f$ is multiplied against in this term is

$$
T(x, k)=\int_{0}^{L_{c} / 2}[U(x+y)-U(x-y)] \sin (2 y k) d y .
$$

Here, $U(x)$ is the electric potential within the device (see below), and $L_{c}$ is the correlation length. The term is nonlinear in $f$ since $U(x)$ depends on $f$ through Poisson's equation.

The final term in the equation, $S(f)$, includes scattering effects in the electron distribution, and its formula is

$$
S(f)=1 / \tau\left[\left(\int_{-\infty}^{\infty} f(x, k) d k / \int_{-\infty}^{\infty} f_{0}(x, k) d k\right) f_{0}(x, k)-f(x, k)\right] .
$$

In this term, $\tau$ is the relaxation time, and $f_{0}(x, k)$ is the equilibrium electron distribution. This is the solution to Eq. (2) when there is no voltage drop applied to the device, (i.e. $V$ $=0$ in Eq. (10) below).

The boundary conditions on $f$ are imposed at the incoming boundaries. For the left side of the device $(x=0)$, this means imposing boundary conditions for $k>0$ since for $k$ $>0$, electrons are moving from left to right. This formula is given by

$$
f(0, k)=4 \pi m^{*} k_{B} T / h^{2} \ln \left(1+\exp \left[1 / k_{B} T\left(h^{2} k^{2} / 8 \pi^{2} m^{*}-\mu_{0}\right)\right]\right) .
$$

For the right side of the device $(x=L)$, this means imposing boundary conditions for $k<$ 0 since for $k<0$, electrons are moving from right to left. This formula is given by

$$
f(L, k)=4 \pi m^{*} k_{B} T / h^{2} \ln \left(1+\exp \left[1 / k_{B} T\left(h^{2} k^{2} / 8 \pi^{2} m^{*}-\mu_{L}\right)\right]\right) .
$$

In the boundary condition formulae, $k_{B}$ is Boltzmann's constant, $T$ is the temperature, $\mu_{0}$ is the Fermi energy at $x=0$, and $\mu_{L}$ is the Fermi energy at $x=L$. Figure 2 gives a graphical representation of the boundary conditions in $(x, k)$ space.

The electrostatic potential, $u(x)$, is the solution to Poisson's equation

$$
d^{2} u / d x^{2}=q^{2} / \varepsilon\left[N_{d}(x)-1 / 2 \pi \int_{-\infty}^{\infty} f(x, k) d k\right]
$$

Here, $q$ is the charge of an electron, $\varepsilon$ is the dielectric permittivity, and $N_{d}(x)$ is the doping profile. The boundary conditions on $u(x)$ are where the voltage drop $V$ comes into the equations. We have

$$
u(0)=0, u(L)=-V
$$

where $V \geq 0$. Once $u(x)$ is solved, then the electric potential is calculated by

$$
U(x)=u(x)+\Delta_{c}(x)
$$




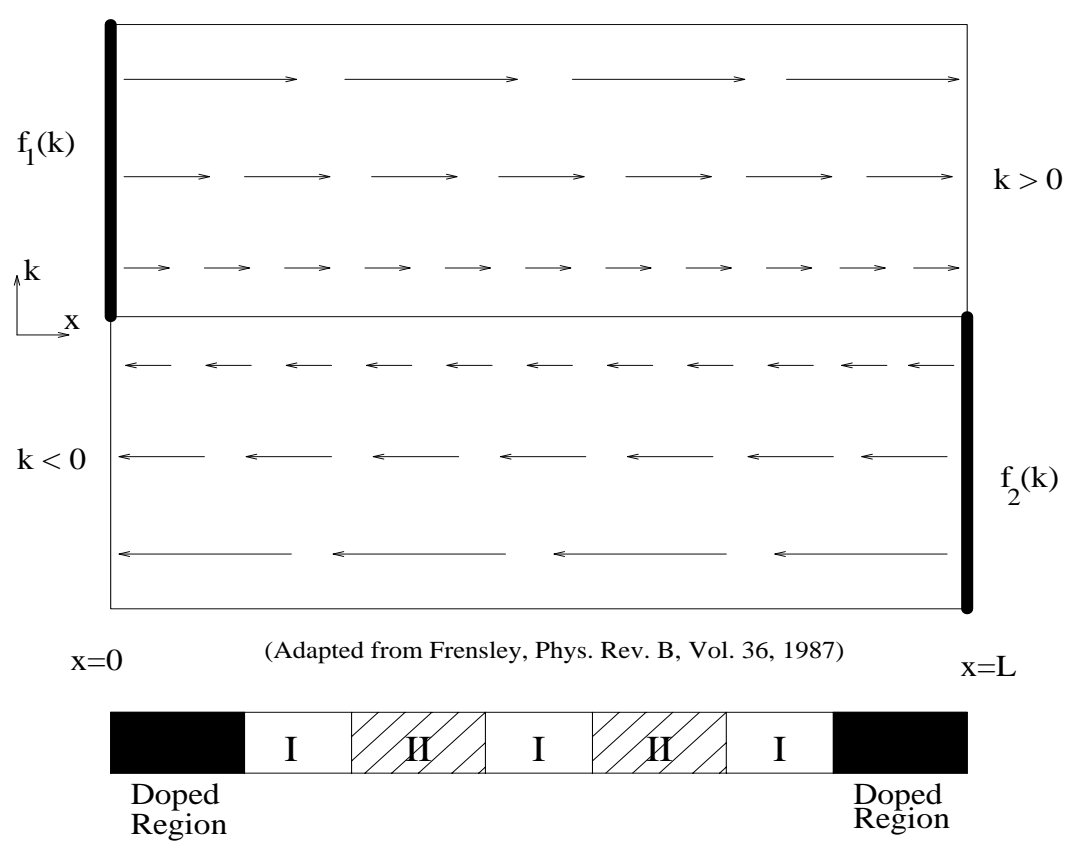

Fig. 2. Boundary conditions on the electron distribution $f(x, k, t)$

Here, $\Delta_{c}(x)$ defines the discontinuous jumps in the electric potential created by the heterojunction of the two different types of semiconductor material.

Finally, to calculate the current density, $j(x, t)$, we use the formula

$$
j(x, t)=h / 2 \pi m^{*} \int_{-\infty}^{\infty} k f(x, k, t) d k .
$$

\section{Discretization}

To numerically solve the Wigner-Poisson equations, we implement a finite difference method. This discretization converts the infinite-dimensional IPDE problem into a finitedimensional nonlinear ODE problem for an approximation of $f$ at the grid points in the $(x$, $k)$ domain. The $x$-domain of $[0, L]$ is discretized with $N_{x}$ equally grid points $x_{i}=(i-$ 1) $\Delta x, i=1,2, \ldots, N_{x}$ and $\Delta x=L /\left(N_{x}-1\right)$. The $k$-domain of $(-\infty, \infty)$ is truncated to $\left(-K_{\max }\right.$, $\left.K_{\max }\right)$ with $N_{k}$ equally spaced grid points $k_{j}=\left(2 j-N_{k}-1\right) \Delta k / 2, j=1,2, \ldots, N_{k}$ and $\Delta k=$ $2^{*} K_{\max } / N_{k}$.

For the kinetic energy term, $K f$, we use an upwind differencing scheme to approximate the spatial derivative term. For $k_{j}>0$, this approximation is given by

$$
K f \approx-h k_{j} / 2 \pi m^{*}\left(-3 f_{i j}+4 f_{i-1, j}-f_{i-2, j} / 2 \Delta x\right)
$$

and for $k_{j}<0$, this approximation is given by 


$$
K f \approx-h k_{j} / 2 \pi m^{*}\left(3 f_{i j}-4 f_{i+1, j}+f_{i+2, j} / 2 \Delta x\right) .
$$

For the integrals in the $P(f)$ and $S(f)$ terms are approximated with a quadrature formula. The approximation for $P(f)$ is given by

$$
P(f) \approx-4 / h \sum_{j=1}^{N_{k}} f_{i j} T\left(x_{i}, k_{j}-k_{j^{\prime}}\right) \Delta k .
$$

The approximation to the kernel function $T\left(x, k-k^{\prime}\right)$ is

$$
T\left(x_{i}, k_{j}-k_{j^{\prime}}\right) \approx \sum_{i^{\prime}=1}^{N_{c}}\left[U\left(x_{i}+x_{i^{\prime}}\right)-U\left(x_{i}-x_{i^{\prime}}\right)\right] \sin \left(2 x_{i^{\prime}}\left(k_{j}-k_{j^{\prime}}\right)\right) \Delta x .
$$

For the scattering term, the quadrature formula gives

$$
S(f) \approx 1 / \tau\left[\left(\sum_{j=1}^{N_{k}} f_{i j} / \sum_{j=1}^{N_{k}} f_{0}\left(x_{i}, k_{j}\right)\right) f_{0}\left(x_{i}, k_{j}\right)-f_{i j}\right] .
$$

Finally, we use a standard three-point center differencing scheme to numerically solve Poisson's equation. This gives for $i=2,3, \ldots, N_{x}-1$

$$
u\left(x_{i-1}\right)-2 u\left(x_{i}\right)+u\left(x_{i+1}\right) / \Delta x^{2}=q^{2} / \varepsilon\left[N_{d}\left(x_{i}\right)-1 / 2 \pi \sum_{j=1}^{N_{k}} f_{i j}\right]
$$

with boundary conditions $u\left(x_{1}\right)=0$ and $u\left(x_{N x}\right)=-V$.

\section{Time-Dependent Simulation}

In the time-dependent simulation, the time-evolution of the electron distribution $f$ is tracked from the ODE using a time-integration technique. In this simulation, the first thing computed is the equilibrium distribution $f_{0}(x, k)$. Next, the voltage drop $V$ is fixed to 0.008 volts, and the electron distribution $f(x, k, t)$ is tracked for 2000 femtoseconds, with the initial condition set to $f(x, k, 0)=f_{0}(x, k)$. As the distribution is being tracked, the current density $j(x, t)$ is computed from Eq. (12). After the 2000 femtosecond simulation, the voltage drop $V$ is increased by 0.008 volts, the final electron distribution from the previous time simulation is set as the initial condition, and the electron distribution is again tracked for 2000 femtoseconds. This process is repeated until a predetermined range of voltages have been processed. 


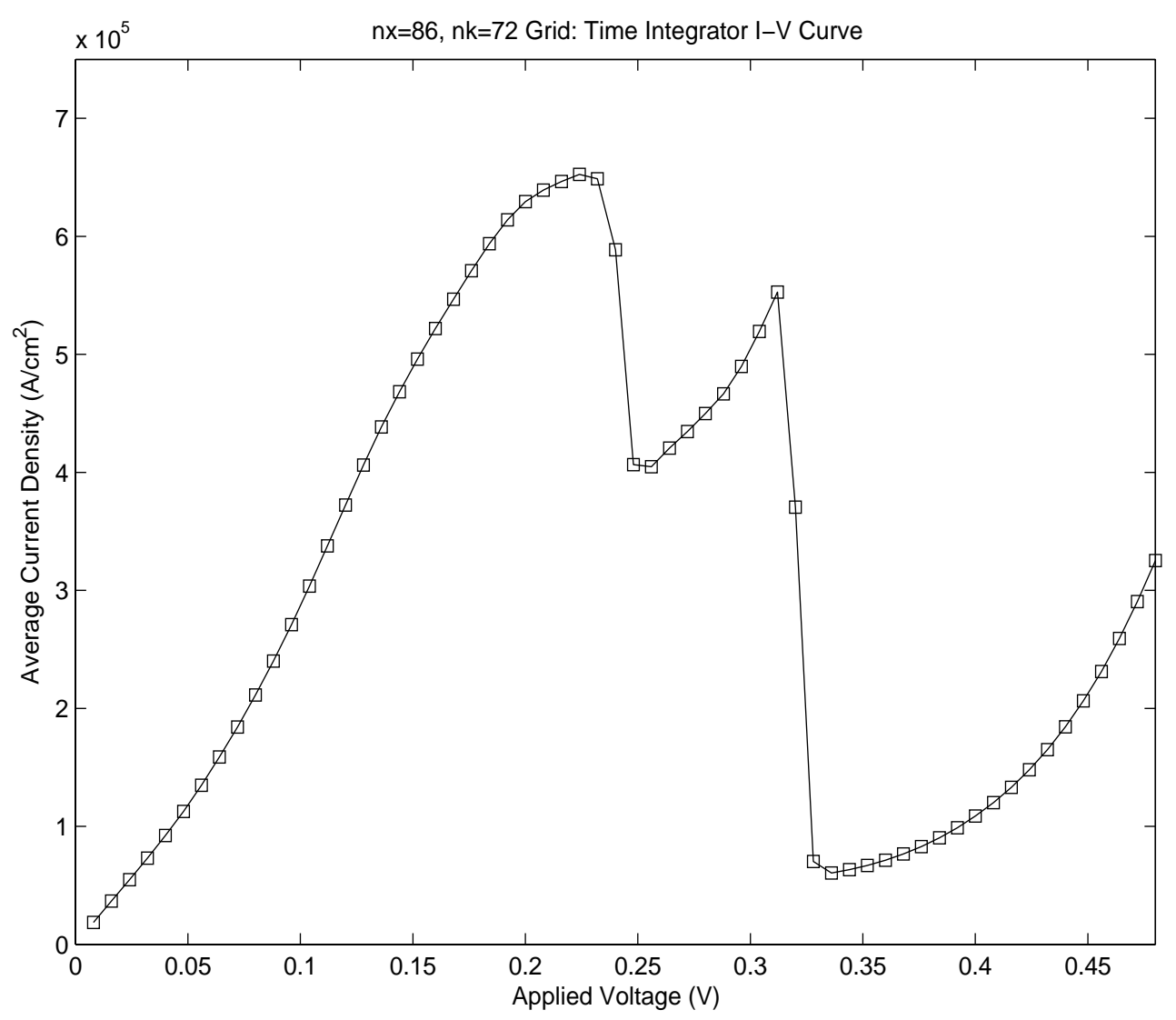

Fig. 3. Time-dependent current-voltage plot

The advantage of the time-dependent simulation is that if current oscillation develops, it will be immediately noticed since the current density is computed as the electron distribution evolves in time. The main disadvantage of this simulation is that it is computationally intensive. The initial time-integration technique took several days to compute the time-evolution of the electron distribution for the various desired voltage drops for coarse grid studies (i.e., $N_{x}=86, N_{k}=72$ or roughly 6,000 unknowns). We have improved upon the time-integration technique (which was previously a semiimplicit Euler's method, but currently is an implicit Adams method ${ }^{8}$ ) and reported on this in previous work ${ }^{9,10}$, but we were still restricted to coarse grid simulation. Figure 3 shows the current-voltage plot generated by the time-dependent simulation. This represents the current at the right-hand side $(x=L)$ after the electron distribution as reached steady-state. If no steady-state was reached but current oscillation occurred, the average value of the current was plotted instead. 


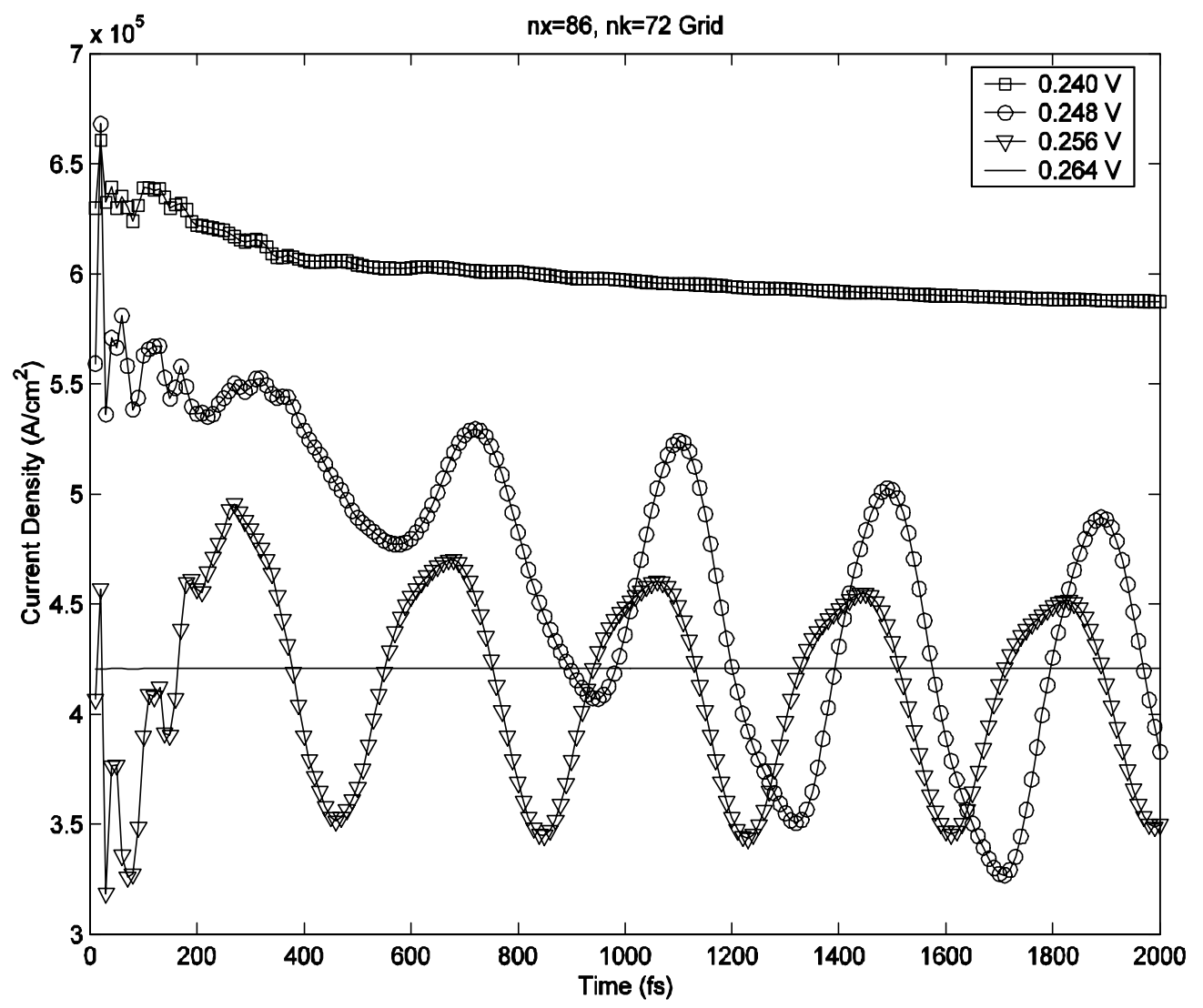

Fig. 4. Voltage drops that create current oscillation within the RTD

Figure 4 shows the voltage drops that lead to current oscillation for the coarse grid. Here, $V=0.248$ volts and $V=0.256$ volts are the two voltage drops where current oscillation is present. If one looks at the current oscillation for $V=0.248$ volts, the period of oscillation appears to be about 400 femtoseconds. This corresponds to a frequency of $2.5 \mathrm{THz}$.

\section{Steady-State Simulation}

The second method of simulation is the steady-state simulation. We now look directly for steady-state electron distributions as the voltage drop is changed. To do this, we solve a nonlinear equation, given by Eq. (2). The advantage of this method is that it is less computationally intensive to calculate the current-voltage curve, with the steady-state simulation taking less than an hour to compute the current-voltage curve. If no current 
oscillation is occurring within the RTD for a given voltage drop $V$, the time-dependent method will converge to the steady-state electron distribution which is much more costly than just directly solving for the steady-state electron distribution. Another advantage of this method is that fine grids can be computed. With this method of simulation, we have computed on the $N_{x}=1024, N_{k}=2048$ grid, which is roughly 2,000,000 unknowns. The disadvantage of this method is that to detect current oscillation from steady-state information, an eigensolve must be performed which is computationally expensive. This is explained in the stability section.

To solve the nonlinear equation for the steady-state electron voltages, we use continuation methods. Continuation methods solve nonlinear equations which depend on a parameter. For example, if we are solving the nonlinear equation $G(z, a)=0$ for $z$, where $z$ is in $R^{\mathrm{m}}$ is our state variable, and $a$, a real number, is our parameter, continuation methods trace out the solution curve $z(a)$, parameterized by $a$, so that $G(z(a), a)=0$. For our particular application, we are solving $W(f, V)=0$, where $f$ is the steady-state electron distribution, and the voltage drop $V$ is the parameter. Numerically, continuation methods generate two sequences: a sequence of parameters $\left\{V^{i}\right\}$ (in our case, voltage drops) with a corresponding sequence of solutions $\left\{f^{i}\right\}$ (in our case, steady-state electron distribution) such that $W\left(f^{i}, V^{i}\right)=0$. To implement the continuation methods numerically, we use the Library of Continuation Algorithms (LOCA) ${ }^{11}$.

LOCA is a part of the Trilinos ${ }^{12}$ framework, Sandia National Laboratories' collection of parallel solver packages. Our implementation of LOCA with our RTD simulator makes use of several other packages in Trilinos, including NOX - the nonlinear solver package, AztecOO - the preconditioned Krylov linear solver package, Anasazi - the eigensolver package, and Epetra - a parallel data structure package. We used a NewtonGMRES algorithm to solve the nonlinear equations, which grouped together the NOX and AztecOO packages, and the Anasazi eigensolver to determine when oscillation onsets. To handle the fine grid simulations, we parallelized the RTD simulator, which involved using the Epetra package.

\section{Stability of Steady-State Solutions}

For the steady-state, since we are not tracking the evolution of the current density over time, we need another method to determine if current oscillation is occurring. Suppose we have the nonlinear ODE $d z / d t=g(z)$ for the state $z$ in $R^{\mathrm{m}}$ and a steady-state solution $z^{*}$, and we want to determine the stability of this steady state solution. One well-known method $^{12}$ would be to compute the eigenvalues of the Jacobian of $g(z)$ evaluated at the steady-state solution $z^{*}$, denoted by $g^{\prime}\left(z^{*}\right)$. If all the eigenvalues have negative real part, then the steady-state $z^{*}$ is stable. If any of the eigenvalues have positive real part, then the steady-state $z^{*}$ is unstable. LOCA interfaces to the Trilinos eigensolver, Anasazi, to compute the leading eigenvalues of this Jacobian matrix as the continuation algorithm finds the steady-state solutions.

For our application, we desire an instability to develop. As the voltage drop $V$ changes, the eigenvalues of the Jacobian matrix at the steady-state electron distribution 
will also change, possibly leading to a change in the stability of the steady-state distribution. This change in stability is known as a bifurcation ${ }^{13}$. In particular, we want to go from a stable steady-state to oscillatory behavior as the voltage drop $V$ is varied. This change in stability is known as a Hopf bifurcation. This bifurcation is characterized by a complex-conjugate pair of eigenvalues of the Jacobian whose real part changes from negative to positive.

The computational challenge with using the eigenvalue method is in detecting the relevant eigenvalues. Anasazi implements an iterative block Arnoldi method for the eigensolve, which quickly locates the eigenvalues with largest magnitude. These eigenvalues are not necessarily the ones we want to find, and therefore, the method may take a while to locate the eigenvalues of interest. One way to handle this problem is to use a spectral transformation to move the eigenvalues of interest so that they have larger magnitudes. Let $J$ denote the Jacobian matrix. The eigenproblem we originally solve is $J z=\lambda z$. The Cayley transformation of the Jacobian matrix is $C=(J-\sigma I)^{-1}(J-\gamma I)$, where $\sigma$ and $\gamma$ are real numbers. The new eigenproblem to solve is $C z=\beta z$. The eigenvectors of $J$ and $C$ are the same, but if $\sigma$ and $\gamma$ are chosen so that $\sigma>0$ and $\gamma=-\sigma$, then the Cayley transformation has the property ${ }^{14}$ that the Jacobian's eigenvalues which have negative real parts are mapped to the interior of the unit-circle, and the Jacobian's eigenvalues which have positive real parts are mapped to the exterior of the unit-circle. Since Anasazi locates eigenvalues of largest magnitude first, when solving the Cayley transformed eigenproblem, it will quickly locate eigenvalues corresponding to the unstable eigenvalues of the Jacobian.

\section{Steady-state Numerical Results}

Figure 5 shows the current-voltage plot generated by the steady-state simulation. This represents the current at the right-hand side $(x=L)$ after the electron distribution as reached steady-state. Note that these results agree with what is produced by the timedependent simulation, except for an additional branch connecting the higher part of the current-voltage curve to the lower part of the current voltage-curve. This branch represents solutions to the Wigner-Equations that are unstable steady-state electron distributions that will not be reached under time-dependent simulation. 


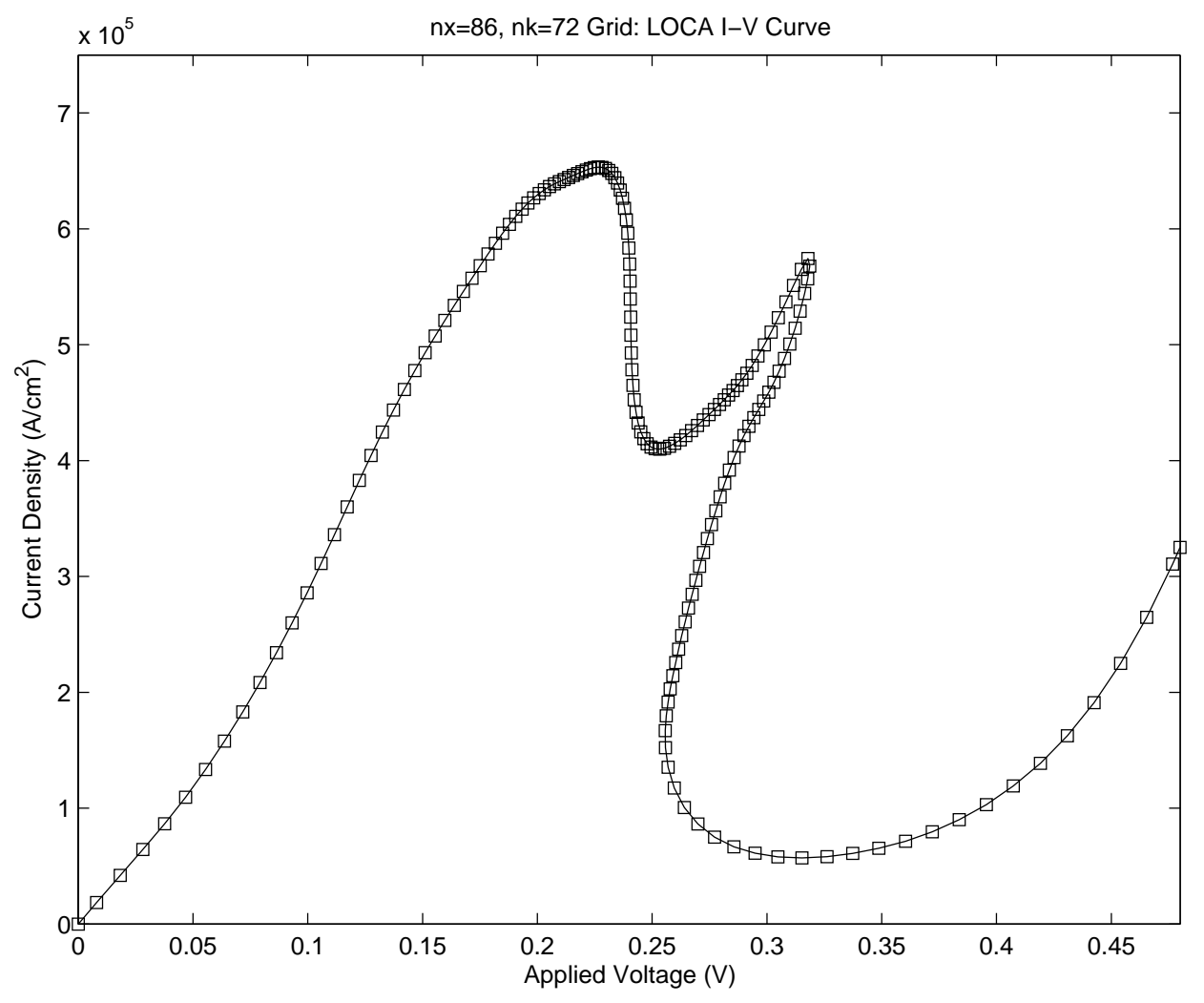

Fig. 5. Coarse grid current-voltage plot from the steady-state simulation

Figure 6 plots the complex-conjugate eigenvalues of the Jacobian matrix responsible for the Hopf bifurcation and onset of current oscillation on the coarse grid. The eigenvalues are tracked, starting at voltage drop $V=0.240$ volts, increasing the voltage drop by 0.002 volts, and ending at $V=0.256$. At $V=0.240$ volts, the real parts of the eigenvalues are negative, so we expect no oscillation. This agrees with what the timedependent simulation produces. Between $V=0.240$ volts and $V=0.242$ volts, the leading eigenvalues' real parts become positive, indicating the onset of oscillation. These real parts stay positive as $V$ increases up to 0.256 volts, where they are about to turn back negative. Therefore, between $V=0.242$ volts and 0.256 volts, we expect oscillation, and as the voltage is further increased past $V=0.256$ volts, we expect the oscillation to stop, and a stable steady-state to return. All of this agrees with what is predicted from the time dependent simulation. Further, at $V=0.248$ volts, the absolute value of the imaginary parts of the eigenvalues is $\omega \approx 0.017$. This corresponds to a period of oscillation of $2 \pi / \omega$ $\approx 370$ femtoseconds, which agrees closely with the time-dependent simulation. 


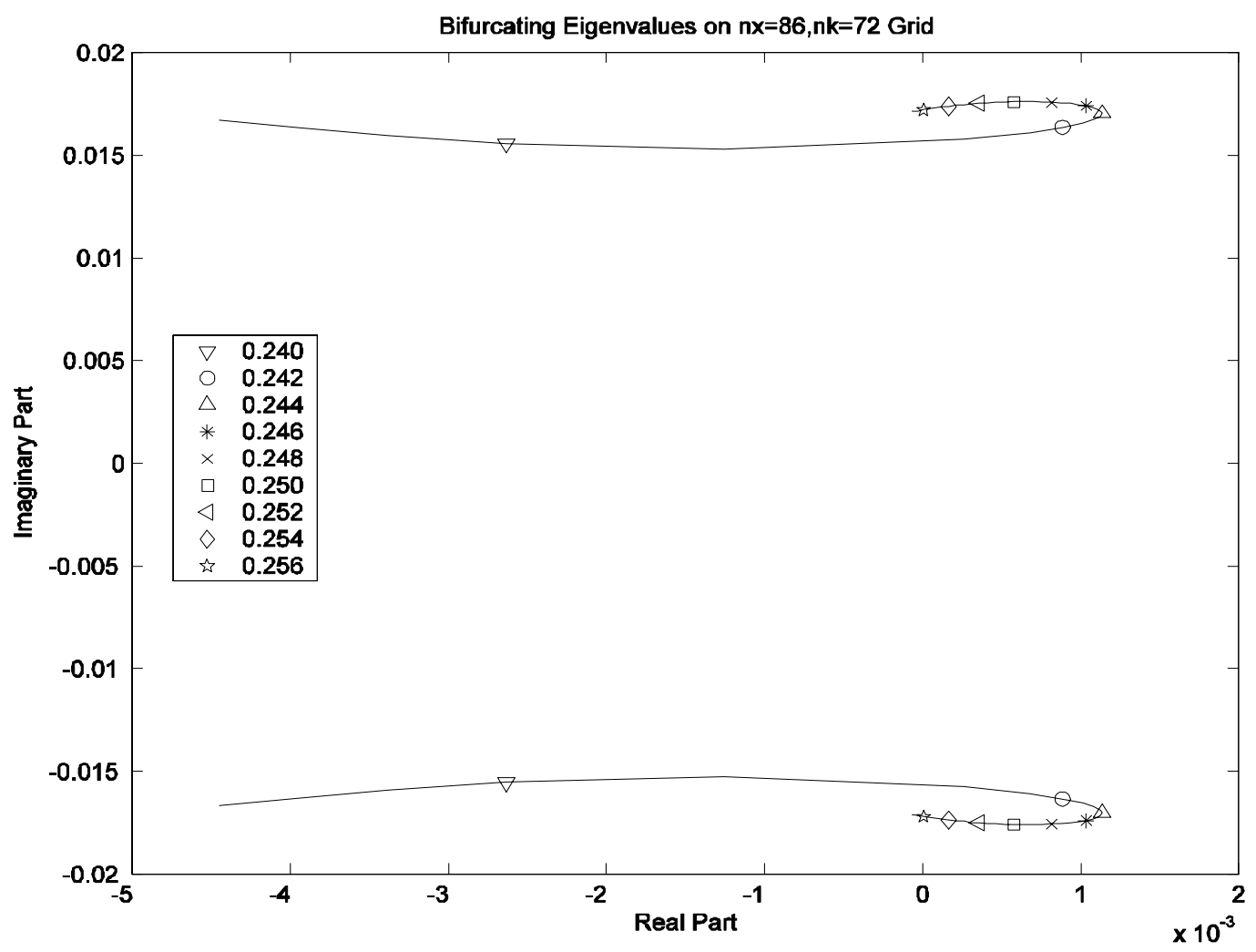

Fig. 6. Complex-conjugate eigenvalues that create oscillation on coarse grid

Figure 7 plots the coarse grid and fine grid current-voltage plots generated by the steady-state method. The fine grid current-voltage curve is lower than the coarse grid current-voltage curve, and the fine grid current-voltage curve has more resonant features than the coarse grid current-voltage curve as evident by the multiple peaks in the fine grid result. These multiple peaks do not match with experimental results, but we note that for the coarse grid result, the correlation length was altered to match the simulated results as much as possible to the experimental results. The correlation length will need to be readjusted for the fine grid simulation as part of our ongoing research.

\section{Conclusions}

We have simulated electron transport within a prototypical nanoscale semiconductor device, a resonant tunneling diode, using the Wigner-Poisson equations in both timedependent and steady-state manners. While the time-dependent method easily detects 


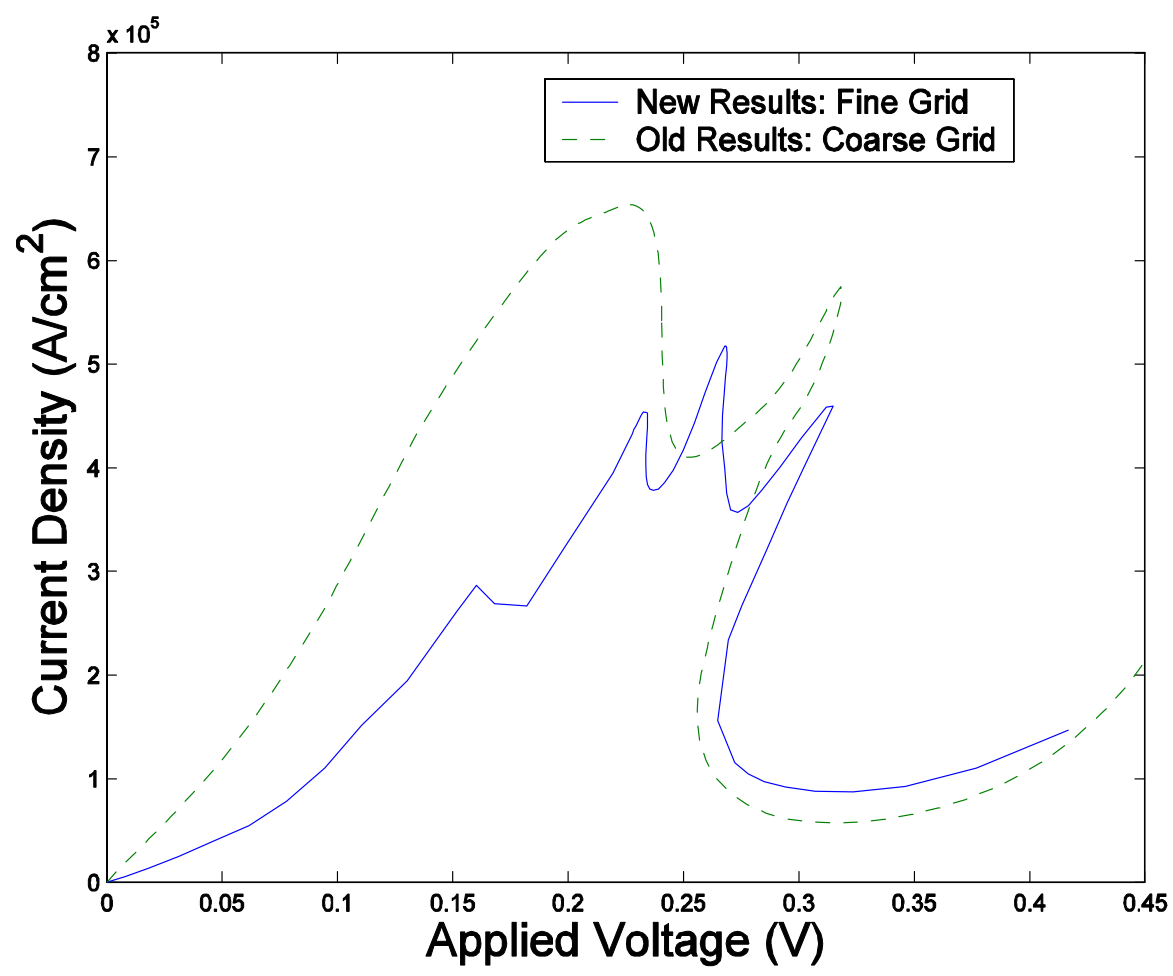

Fig. 7. Comparison of coarse grid and fine grid current-voltage plots

current oscillation, its computational cost makes this method feasible for only coarse grids. In contrast, the steady-state method can handle fine grids and more quickly generates the current-voltage curve of the RTD than the time-dependent methods, but detection of current oscillation requires a computationally intensive eigensolve. The Cayley transformation eases the computational burden of the eigensolve, and current research is focusing on finding a correlation length that makes the current-voltage curve produced on the fine grids match with experimental results and using the Cayley transformation to locate the onset of current oscillation on the fine grids.

\section{Acknowledgements}

This research is supported by the U.S. Army Research Office's Multidisciplinary University Research Initiative (MURI) program on "The Science and Technology of Nano/Molecular Electronics: Theory, Simulation, and Experimental Characterization". Sandia is a multiprogram laboratory operated by Sandia Corporation, a Lockheed-Martin Company, for the United States Department of Energy under contract DE-AC0494AL85000. 


\section{References}

1. G. E. Moore, Cramming more components into integrated circuits, Electronics. 38(8), April 19, 1965.

2. E. Wigner, On the quantum correction for thermodynamic equilibrium, Phys. Rev. 40, 749-759 (1932).

3. T. C. L. G. Sollner, W. D. Goodhue, P. E. Tanndenwald, C. D. Parker, and D. D. Peck, Resonant tunneling through quantum wells at frequencies up to $2.5 \mathrm{THz}$, Appl. Phys. Lett. 43, 588-590 (1983).

4. T. C. L. G. Sollner, P. E. Tanndenwald, D. D. Peck, and C. D. Parker, Quantum well oscillators, Appl. Phys. Lett. 45, 1319-1321 (1984).

5. W. R. Frensley, Wigner-function model for a resonant tunneling semiconductor device, Phys. Rev. B 36, 1570-1580 (1987).

6. P. Zhao, D. L. Woolard, H. L. Cui, Multisubband theory for the origination of intrinsic oscillations within double-barrier quantum well systems, Phys. Rev. B 67 (2003).

7. D. L. Woolard, P. Zhao, H. L. Cui, THz-frequency intrinsic oscillations in double-barrier quantum well systems, Physica B 314, 108-112 (2002).

8. A. Quarteroni, R. Sacco, and F. Saleri, Numerical mathematics, Volume 37 of Text in Applied Mathematics, Springer-Verlag, New York (2000).

9. C. T. Kelley, D. L. Woolard, P. Zhao, M. Kerr, and M. S. Lasater, Parallel-platform based numerical simulation of instabilities in nanoscale tunneling devices, Proceedings of $20022^{\text {nd }}$ IEEE Conference on Nanotechnology, 417-421 (2002).

10. M. S. Lasater, P. Zhao, C. T. Kelley, and D. L. Woolard, Numerical tools for the study of instabilities within the positive-differential-resistance regions of tunneling devices, Proceedings of $20033^{\text {rd }}$ IEEE Conference on Nanotechnology, 390-393 (2003).

11. A. G. Salinger, N. M. Bou-Rabee, R. P. Pawlowski, E. D. Wilkes, E. A. Burroughs, R. B. Lehoucq, and L. A. Romero, LOCA 1.0 Library of continuation algorithms: theory and implementation manual, Technical Report SAND2002-0396, Sandia National Laboratory (2002).

12. M. Heroux, R. Bartlett, V. Howle, R. Hoekstra, J. Hu, T. Kolda, R. B. Lehoucq, K. Long, R. P. Pawlowski, E. Phipps, A. G. Salinger, H. Thornquist, R. Tuminaro, J. Willenbring, and A. Williams, An overview of Trilinos, Technical Report SAND2003-2927, Sandia National Laboratory (2003).

13. E. Kreyszig, Elements of applied bifurcation theory, Volume 112 of Applied Mathematics Sciences, Springer-Verlag, New York (1998).

14. E. A. Burroughs, L. A. Romero, R. B. Lehoucq, and A. G. Salinger, Linear stability of a flow in a differentially heated cavity via large-scale eigenvalue calculations, Int. J. Num. Meth. Heat and Fluid Flow 14(6), 803-822 (2004). 